\title{
Tachykinin-Immunoreactive Neurons in Developing Feline Neostriatum: Somatodendritic Morphogenesis Demonstrated by Combined Immunohistochemistry/Golgi Impregnation-Gold Toning
}

\author{
Robin Fisher Marea Boylan \\ Developmental and Molecular Neuroscience Group, Intellectual Development and Disabilities Research Center, \\ Neuropsychiatric Institute, School of Medicine, University of California, Los Angeles, Calif., USA
}

\section{Key Words}

Neuronal development $\cdot$ Basal ganglia $\cdot$ Medium spiny neurons $\cdot$ Neuropeptides $\cdot$ Golgi staining $\cdot$ Double labeling

\begin{abstract}
This investigation was designed to survey and characterize the development of a key link between chemically mediated neurotransmission and neuronal cytoarchitecture in mammalian basal ganglia. Peroxidase immunohistochemical and Golgi impregnation/gold toning methods were combined to doubly label the tachykinin neuromodulator signature and somatodendritic structure of neostriatal neurons in late fetal, postnatal and adult cats. The results supported 3 conclusions of considerable significance. (1) Colocalization of immunohistochemical and Golgi impregnation/gold toning labels is a feasible, rational and productive means to identify and determine the somatodendritic morphogenesis of tachykinin neurons. (2) The application of this method to developing feline neostriatum demonstrates directly that the principal tachykinin cells are medium-sized spiny neurons, which undergo progressive growth and elaboration of cell bodies, dendritic arbors and dendritic spines during the late fetal and postnatal periods. (3) There is a strong but incom-
\end{abstract}

\section{KARGER}

Fax +4161306 1234

E-Mail karger@karger.ch

www.karger.com (c) $2011 \mathrm{~S}$. Karger AG, Basel

$0378-5866 / 11 / 0331-0075 \$ 38.00 / 0$

Accessible online at:

www.karger.com/dne plete concordance between tachykinin and medium-sized spiny neuronal phenotypes, because a minor variant of medium-sized spiny neurons and rare subgroups of mediumand large-sized sparse spiny neurons also show the tachykinin neuromodulator signature. Taken together, these results suggest that neostriatal neurons show an early commitment to heterogeneous tachykinin phenotypes, although the full and final expression of their somatodendritic characteristics coincides with synaptogenesis.

Copyright $\odot 2011$ S. Karger AG, Basel

\section{Introduction}

Determination of the nexus between chemically mediated neurotransmission and neuronal cy toarchitecture is a central and persistent problem in modern neuroscience. While technically difficult to resolve in substantial detail, the issue is of particular interest when the signaling agent is produced both before and after the age-related assembly of synaptic junctions. The 3 main members of the tachykinin family of neuropeptides (substance $\mathrm{P}$, neurokinin A and neurokinin B) provide an example of an important functional shift related to synaptogenesis, as 
shown by their initial trophic and subsequent modulatory roles in the developing basal ganglia of mammals. In immature rats and cats, tachykinins show a pattern of early, progressive expression in numerous small to medium neurons in the neostriatum (caudate nucleus, putamen and, when separate, their intervening cell bridges) during middle and late fetal periods, prior to their perinatal establishment of significant synaptic connectivity via local axon collaterals or descending (i.e. striatonigral) axon projections $[1,2]$. The morphogenetic properties of these cells are largely uncharted, and their characterization is the first objective of the present study. In mature rats and cats, about half of neostriatal neurons encode and accumulate tachykinins [3-5]. Usually described as medium cells, they are similar to the principal and most frequent medium spiny neurons found in all parts of the neostriatum [3-9]. However, these cells may have heterogeneous structural identities because instances of large and medium tachykinin immunoreactive neurons, probably of infrequent aspiny phenotypes, have also been reported $[10,11]$. The only previous direct test of structural diversity among neostriatal tachykinin cells is somewhat inconclusive, due to its exclusive colocalization of immunoreactive and somatodendritic (Golgi impregnation/ gold toning, GG) labels in a very small complement of medium spiny neurons (a low yield of 33 cells obtained from 3 adult cats and 2 ferrets) [12]. This informative but underutilized method has been modified substantially to increase the yield of doubly labeled cells, and thus to delineate the full range of cytoarchitectural phenotypes of tachykinin neurons in neostriatum as a 2 nd objective in the present study. The investigation is performed in an unusual species, cats, in order to take advantage of the compartmentalized regional organization of the carnivore forebrain, and to provide the adequate supply of tissue necessary to assess the validity of label patterns obtained from each case in a developmental time series with prenatal and postnatal age levels. The outcomes demonstrate the strengths and limitations of this approach for the morphogenetic analysis of neurons identified by their neurochemical signature.

\section{Methods}

The colocalization of 2 labels that simultaneously define neuromodulator (tachykinin immunoreactivity, TI) and somatodendritic (GG) neuronal phenotypes in neostriatum was tested in 10 cats at the following ages: F52 (fetal day $52 ; \mathrm{n}=1$; feline gestation $=65$ days), F56 $(n=1)$, P1 (postnatal day $1 ; n=2)$, P30 $(n=2)$ and adult $(1-3$ years postnatally; $\mathrm{n}=4)$. All cats were housed in the UCLA School of Medicine vivarium, and procedures conducted as per the 'National Institutes of Health Guide for the Care and Use of Laboratory Animals'. Fetuses were surgically removed from pregnant queens anesthetized with halothane. All anatomical cases were deeply anesthetized with barbiturate $(100 \mathrm{mg} / \mathrm{kg}$ body weight Nembutal) and perfused transcardially with $0.87 \%$ saline rinse followed by fixative ( $4 \%$ paraformaldehyde in $0.1 \mathrm{M}$ phosphate-buffered $0.87 \%$ saline; $\mathrm{pH}=7.4 ; 4^{\circ} \mathrm{C}$ ). The fixative included $0.5 \% \mathrm{ZnCrO}_{4}$, a method modification that enhanced the density of perisomatic immunolabeling. Within $1 \mathrm{~h}$ from initiation of fixation, brains were blocked into hemispheres, surrounding dorsal pallium and septae were removed from lateral ventricles, and specimens were embedded in agar. Coronal plane tissue sections of $100 \mu \mathrm{m}$ thickness were cut by a Vibratome into $0.1 \mathrm{M}$ Tris-buffered $0.87 \%$ saline; $\mathrm{pH}=7.4 ; 4^{\circ} \mathrm{C}$. Each case provided a series of at least 24 sections, with neostriatal samples at head, body and tail of the caudate nucleus.

The TI label was produced by peroxidase-antiperoxidase methods as detailed previously $[2,13]$. After conventional pretreatments excluding detergents in order to minimize tissue disruption, sections were incubated in rat IgG monoclonal primary antibody directed against substance P [14] (Pelfreez; 1:1,000 optimal dilution; $24 \mathrm{~h} ; 4^{\circ} \mathrm{C}$ ). This antibody crossreacts with substance $\mathrm{P}$, neurokinin A and neurokinin B. Rabbit antirat IgG was the secondary antibody (Pelfreez; 1:80 optimal dilution; $30 \mathrm{~min}$; $25^{\circ} \mathrm{C}$ ) followed by rat IgG-peroxidase-antiperoxidase complex (Sternberger-Meyer; 1:100 optimal dilution; $30 \mathrm{~min} ; 25^{\circ} \mathrm{C}$ ). TI label was generated from 3,3'-diaminobenzidine chromogen by glucose oxidase-coupled peroxidase histochemical methods [15] (55 min; $34-37^{\circ} \mathrm{C}$; no cobalt enhancement).

The GG label was subsequently produced by an initial 'singlesection' Golgi impregnation method [16] followed by gold toning [17]. Each section was postfixed in $0.2 \% \mathrm{OsO}_{4}$ (Tris-buffered saline; $5 \mathrm{~min}$ ), $10 \% \mathrm{~K}_{2} \mathrm{Cr}_{2} \mathrm{O}_{7}$ (Tris-buffered saline; $24 \mathrm{~h} ; 25^{\circ} \mathrm{C}$ ), assembled between a glass coverslip and slide, with cement at coverslip corners for fluid access at section edges, not faces, and immersed in $4 \% \mathrm{AgNO}_{3}\left(\mathrm{H}_{2} \mathrm{O} ; 24 \mathrm{~h} ; 25^{\circ} \mathrm{C}\right.$; dark storage). After $\mathrm{Ag}_{2} \mathrm{CrO}_{4}$ impregnation, each assembly was transilluminated with a fiberoptic illuminator, with maximum output $1 \mathrm{~cm}$ from the section face, and reflective backlighting (30-60 s exposure/face) [18]. This method modification photoreduced, at least in part, silver chromate to metallic silver, which promoted a fine-grained perisomatic distribution of metallic gold for the GG label in translucent cell bodies. Assemblies were dismantled, and sections were immersed in $0.01 \%$ gold(III) chloride solution $\left(\mathrm{HAuCl} \cdot 4 \mathrm{H}_{2} \mathrm{O} ; 55\right.$ $\min ; 25^{\circ} \mathrm{C}$; dark storage). This reduction-oxidation reaction deposited metallic gold particles onto silver chromate and metallic silver, which were then removed by $1 \%$ sodium thiosulfate treatment. Finally, sections were dehydrated in alcohols, cleared in xylenes and coverslipped with DePeX ${ }^{\mathrm{TM}}$ mountant.

Differential labeling of 'experimental' and 'control' sections obtained from each case established methodological specificities for TI and GG labels by their selective omission. The TI 'singledeletion' control was replacement of primary antibody by nonimmune rat serum. Additional specificity tests for this antibody were provided [2]. The GG 'single-deletion' control was replacement of silver nitrate by water diluent. The TI + GG 'double-deletion' control applied both single-deletion conditions. Morphological distributions of specific TI and GG labels in control and experimental sections were assessed by light microscopy with a 

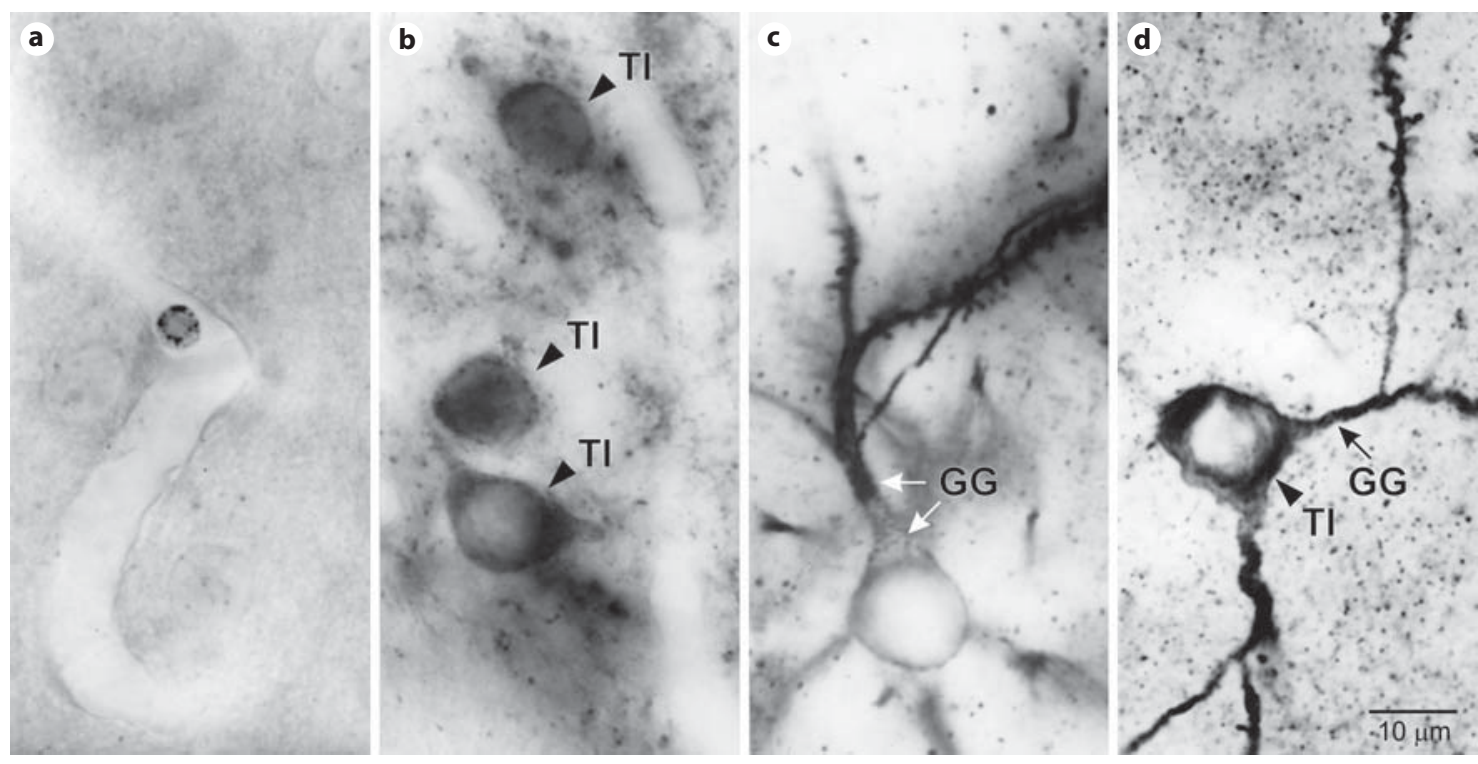

Fig. 1. Photomicrographic series $(100 \times$ oil immersion planoapochromatic objective, n.a. $=1.3$, brightfield transmission illumination) of label deletion control outcomes in adult feline neostriatum. a 'Double-deletion' control section with selective elimination of both TI and GG labels. The nonspecifically labeled element in the blood vessel is an erythrocyte fragment. b GG 'single-deletion' control section with selective elimination of GG and spe- cific labeling of medium TI neurons (arrowheads). c TI 'singledeletion' control section with selective elimination of TI and specific labeling of GG (arrows to somatic and dendritic locations) in a medium spiny neuron. d 'Experimental' section demonstrating a doubly labeled medium spiny TI (arrowhead) + GG (arrow) neuron. Scale $=10 \mu \mathrm{m}$.
Zeiss Ultraphot IIIB by use of brightfield transmission and differential interference contrast illuminations. Structural details of singly and doubly labeled neurons were recorded by photomicroscopy and camera lucida drawings. These data were compared across the developmental sequence, with ancillary observations obtained as needed, from kitten reference materials in our collection.

\section{Results}

\section{Label Foundations}

Both TI and GG labels exhibited reasonable method specificity, and were additive in combination for all 10 tested cases. Neurons defined by profiles with diameters $\geq 7 \mu \mathrm{m}$ were the locus for these tests. In TI + GG doubledeletion control sections, neither label was apparent (fig. 1a). Amorphous, slightly yellow background stain permeated the section thickness due to osmication/chromation. Erythrocytes contained diaminobenzidine chromogen due to residual oxidase activity. In GG single-deletion control sections, TI but not GG single label consisted of perisomatic, reddish brown material in neurons sited $<15 \mu \mathrm{m}$ from both section faces (fig. 1b). TI also re- sided in small ( $\leq 1 \mu \mathrm{m}$ diameter) puncta and thin processes of local axonal and dendritic arbors. In TI singledeletion control sections, GG but not TI single label consisted of perisomatic, translucent gray metal deposits continuous with denser gray-black metal deposits in dendritic arbors and specializations, in neurons sited throughout the entire section thickness (fig. 1c). GG also resided in scattered puncta, processes, glial cells and endothelial cells.

In experimental sections with successful Golgi impregnation of neostriatal neurons $(40-50 \%$ from each tested case at all ages), TI + GG labels were colocalized in doubly labeled cells when perisomatic TI was contained within a translucent GG cell body continuous with metal-impregnated dendrites in neurons sited $<15 \mu \mathrm{m}$ from section faces (fig. $1 \mathrm{~d}$ and 2, color insets). In these cells, translucent gaps of somatic and proximal dendritic (1storder segments) GG label were occasionally found between the chromatic accumulation of somatic TI label and the denser metallic label of more distal dendritic (1storder segments) GG label. Consistent with singly labeled cell bodies in single-deletion control sections, TI and GG neurons, as well as unlabeled neurons, were also found. 


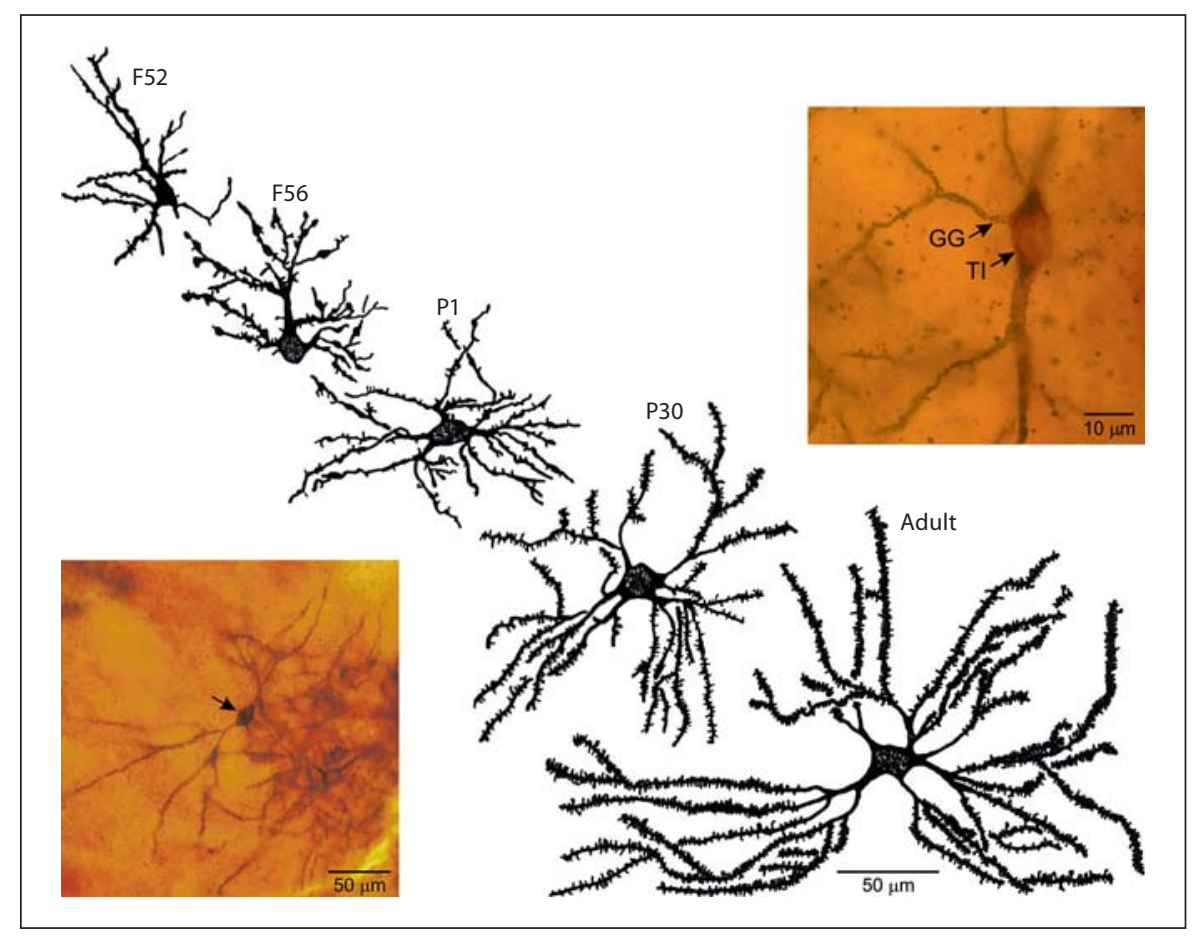

Fig. 2. Camera lucida drawing series demonstrating the development (ages F52 to adult) of dendritic arbors for the principal phenotype of medium spiny TI + GG neurons in feline neostriatum. Note the age-related growth of dendritic arbors, prenatal increase in dendritic branches and increase in dendritic spine densities. Scale $=50 \mu \mathrm{m}$. Color inset lower left: 'low' resolution $(25 \times$ dry planoapochromatic objective, n.a. $=0.65$, brightfield transmission illumination) color photomicrograph of a doubly labeled TI + GG medium spiny neostriatal neuron (arrow) from an 'experimental' section of a P30 kitten. Scale $=50 \mu \mathrm{m}$. In this initial scanning phase of our detection procedure, singly labeled TI neurons are barely discerned due to the extended optical depth of field, and doubly labeled neurons infrequently display the dense accu-

No TI + GG glial or endothelial cells were seen, although GG cells of these types were apparent. TI + GG puncta and processes were recognized but not detailed, due to the difficult optical resolution of diffuse, intermingled labels in such small profiles.

TI + GG labels were precisely identified by correlative light/electron microscopy in a doubly labeled cell (a medium spiny neuron), which colocalized diffuse electrondense material (TI) and dispersed electron-opaque particles (GG) (data not shown). This cell had synaptic junctions, but its fine structure was compromised by inadequate fixation. TI and GG labels were accurately identified by light microscopy in both singly and doubly labeled cells (fig. 2, color insets). Chromatic TI label was mulation of TI label shown by this example. Color inset upper right: 'high' resolution $(100 \times$ oil immersion planoapochromatic objective, n.a. $=1.3$, brightfield transmission illumination) color photomicrograph of a doubly labeled TI + GG medium spiny neostriatal neuron from an 'experimental' section of a P30 kitten. Scale $=10 \mu \mathrm{m}$. In this subsequent confirmation phase of our detection procedure, a narrow depth of optical field is used to definitively colocalize perisomatic reddish brown material (TI label at arrow) and dendritic gray-black metal deposits (dense GG label at arrow; perisomatic GG label is dispersed and translucent) within an individual neuronal cell body and its processes, an outcome required for all doubly labeled TI + GG neurons. Spines are typically infrequent along proximal dendritic segments of such cells.

optically eliminated at a narrow band of red-yellow (i.e. reddish brown) wavelengths by running continuous interference monochromator. Nonchromatic GG label reliably formed diffuse perisomatic versus dense dendritic metal deposits [18]. In both subcellular sites, metal particles were opaque, nonreflective by incident illumination and impervious to selective elimination by monochromator. Fetal neostriatum presented a technical limitation because unembedded, free-floating and gold-toned sections were brittle. They tended to crack when mounted, which curtailed dendritic continuity of TI + GG and GG neurons. Consequently, double labeling was not pursued at ages $<$ F52, even though TI and Golgi impregnations were separately obtained as early as F30. 

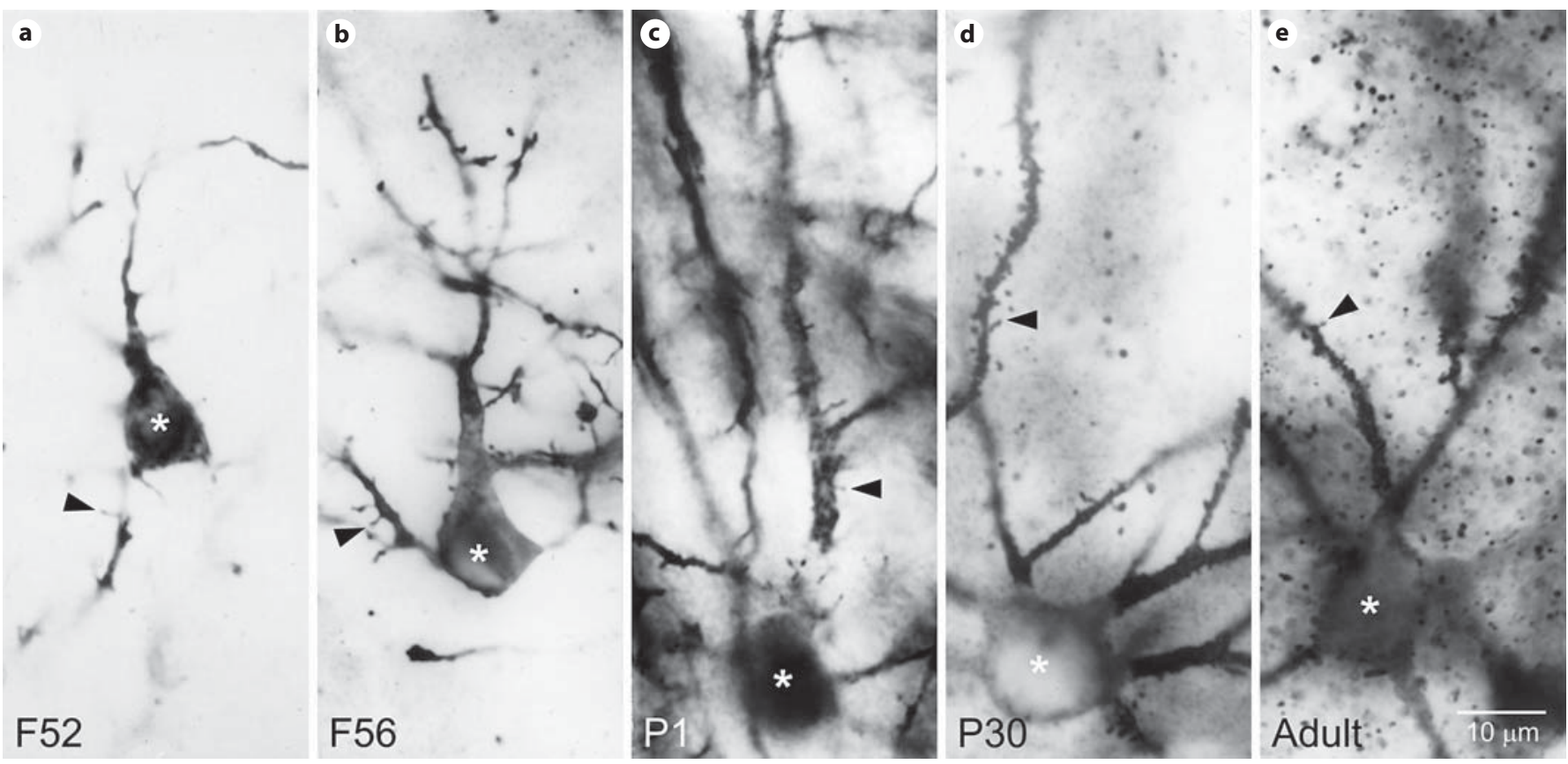

Fig. 3. Photomicrographic series $(100 \times$ oil immersion planoapochromatic objective, n.a. $=1.3$, brightfield transmission illumination) demonstrating the development (ages F52 to adult) of somatodendritic characteristics for the principal phenotype (and its minor variant) of medium spiny TI + GG neurons in feline neostriatum. Doubly labeled cell bodies are shown by asterisks, and dendritic spines are shown by arrowheads. Note the age-related growth of cell bodies and dendritic spine densities. Scale $=10 \mu \mathrm{m}$.

Fig. 4. Photomicrographic series $(100 \times$ oil immersion planoapochromatic objective, n.a. $=1.3$, brightfield transmission illumination) demonstrating somatodendritic characteristics of the 3 phenotypes of TI + GG neurons in adult feline neostriatum. a-c Doubly labeled cell bodies are shown by asterisks, with somatodendritic TI + GG phenotypes (a medium spiny, b medium aspiny, c large aspiny) identified for each panel. $\mathbf{d}-\mathbf{f}$ Dendritic spines on distal dendritic branches (orders $>3$ ) for each of the phenotypes listed above are shown by arrowheads. Scale $=10 \mu \mathrm{m}$.
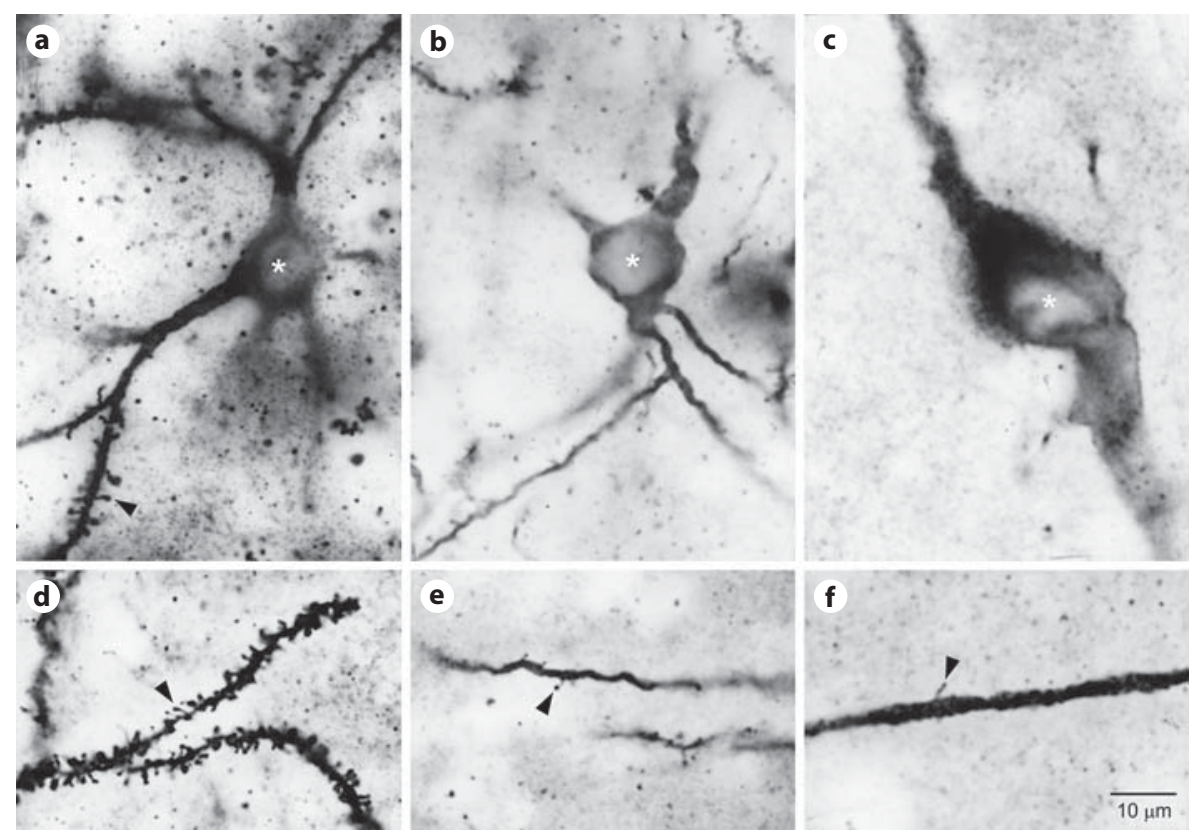
Fig. 5. Camera lucida drawings demonstrating the distinct somatodendritic medium spiny (predominant), large aspiny (rare) and medium aspiny (rare) phenotypes of doubly labeled TI + GG neurons in adult feline neostriatum. Scale $=50 \mu \mathrm{m}$.

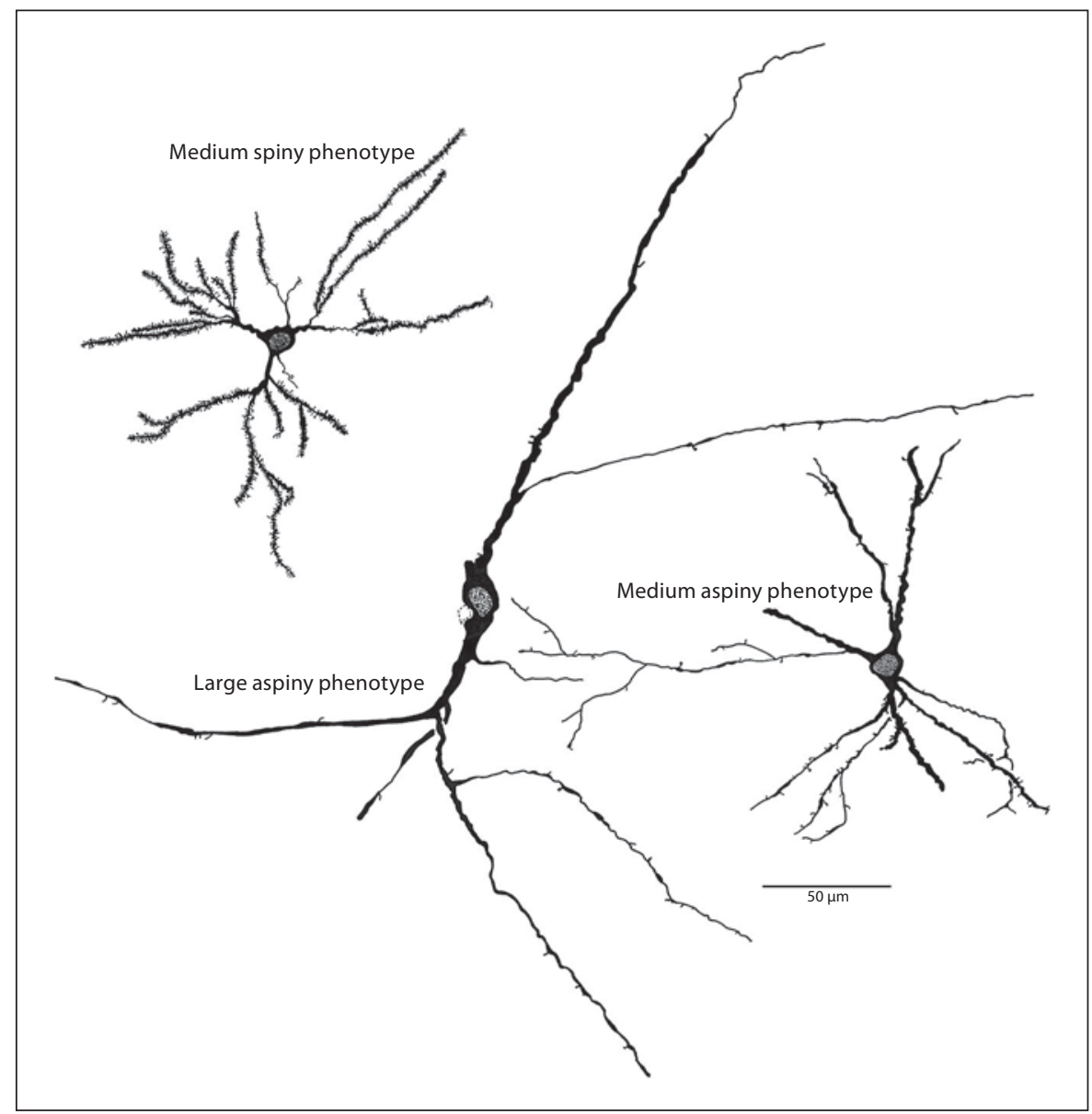

\section{Morphogenesis of Medium Spiny TI + GG Neurons}

Observations were obtained from $>1,500 \mathrm{TI}+\mathrm{GG}$ (i.e. doubly labeled) neurons (25-250 cells/case; F52/56 cases yielded about 25 cells each, and older cases yielded at least 100 cells each; F52/56 cases were pooled as nearreplicates in a fetal age group for most of the analysis below). In order to classify somatodendritic phenotypes, each neuron in this sample had at least 3 branch orders in 1 dendrite continuous with a doubly labeled cell body. At all ages, TI + GG neurons were settled in all parts of the neostriatum, with no regional differences. This distribution matched the progressively expanded mantle zone of developing neostriatum. TI + GG and TI (i.e. singly labeled) neurons were not found in transient, progressively contracted subventricular and intermediate zones.

The principal TI + GG neurons observed in neostriatum on and after F52 (all but 10 of the total double-labeled sample) were medium spiny neurons with somatic diameters from 8 to $18 \mu \mathrm{m}$, and maximum spine densities on distal dendritic shafts (i.e. 3rd- and higher-order branch- es; fig. 2, color insets, 3a-e, and 4a). Phenotypic classification was least certain at F52, when spine densities were lowest in all branch orders, and each neuron's principal dendrites had heterogeneous maturational states.

The TI + GG medium spiny neurons had 1 major phenotype and a minor variant evident on and after F56. Nuclei were sometimes lost or obscured by overlaid TI label (fig. 3c, e), but many TI + GG neurons retained nuclear profiles, which were spherical, central and predominant within the cell bodies. In the major phenotype (70\%), nuclear profiles were smooth with homogeneous content, somatic and proximal dendritic spines were infrequent or absent, and initial segments of a single, thin axon emerged, when detected, from cell bodies (fig. $3 \mathrm{a}$ and $4 \mathrm{a}$ ). In the minor variant $(30 \%)$, dark creases or folds (TI label in partly enclosed, extra-perisomatic cytoplasm) overlaid or invaginated nuclear profiles, somatic and proximal dendritic spines were more frequent, and initial axon segments emerged, when detected, from proximal dendrites (fig. $1 \mathrm{~d}$ and $3 \mathrm{~d}$ ). 
The major phenotype and minor variant of TI + GG medium spiny neurons had comparable patterns of progressive somatodendritic morphogenesis after F52/56 (fig. 2 and 3). Maturation tended to be more advanced in first-formed, ventrolateral parts of neostriatum before P30. Morphogenesis of distinct structural characteristics was best discerned in composite camera lucida drawings of representative TI + GG neurons from each age group (fig. 2), which brought dendritic arbors into the same apparent focal planes as their cell bodies. It was noted that single-section Golgi impregnation revealed only $100-\mu \mathrm{m}$ thick 'slices' of dendritic arbors of TI + GG neurons, with cell bodies only in $15-\mu$ m-thick immunoreactive section faces. Under these constraints, relatively greater portions of the full dendritic arbors were observed in younger smaller cells than in older larger cells, a systematic bias likely to underestimate the true extent of their growth.

Somatic growth was shown by a 2 -fold increase in average intact cell body diameters ( 8 vs. $16 \mu \mathrm{m}$ in F52/56 kittens and adults). There was a comparable 2-fold increase in average intact nuclear diameters (5 vs. $10 \mu \mathrm{m}$, respectively). Modeled as simple spherical profiles $(\mathrm{V}=$ $\left.4 / 3 \pi r^{3}\right)$, these values yielded 8 -fold volume increases for perisomatic cytoplasm (202 vs. 1,621 $\mu^{3}$ ) and nucleoplasm (66 vs. $523 \mu \mathrm{m}^{3}$ ).

Dendritic process growth was shown by at least a 3 -fold increase in average maximum diameters of dendritic fields (75 vs. $250 \mu \mathrm{m}$ in F52/56 kittens and adults). Outgrowth by elongation occurred throughout the study period, although dendritic shafts had expanded to nearuniform calibers of $\geq 1-2 \mu \mathrm{m}$ by P30. Outgrowth by branching occurred between F52/56 and P1, when average maximum branches of most intact principal dendrites increased from 4 to 7 orders. These neurons were multipolar, but 1-2 1st-order dendritic branches were usually gained between F52/56 and P1. Dendritic growth cones were infrequent prenatally and absent postnatally.

Dendritic spine growth was shown by a 100 -fold increase in spine density on distal dendritic shafts (3rd order and higher) between F52/56 and adulthood. Spine density decreased with age on proximal dendrites (1st and 2nd orders) due to shaft elongation. For the major phenotype, infrequent spines on 1st-order dendrites were largely eliminated by $\mathrm{P} 1$.

\section{Unusual Somatodendritic Phenotypes of TI + GG Neurons}

In accord with the classification of mature Golgi-impregnated feline neostriatal neurons [19], most (>95\%) singly and doubly labeled GG neurons had the medium spiny somatodendritic phenotype. However, each case also presented low frequencies of singly labeled GG neurons with small, medium and large cell bodies and sparse ('aspiny') dendritic spines. As shown in figure $4 \mathrm{a}-\mathrm{f}$, infrequent cells with 2 of these phenotypes were also doubly labeled in some, but not all cases at ages $\geq P 1$, and were obviously different from the principal and variant medium spiny TI + GG phenotype.

Five medium aspiny TI + GG neurons from 4 cases were spherical multipolar cells with somatic diameters of 12-16 $\mu \mathrm{m}$, smooth dendritic shafts with calibers of 1-2 $\mu \mathrm{m}$, and sparse dendritic spines dispersed throughout the dendritic arbors. Their dendritic arbors, including branch patterns, segment lengths and shaft calibers, matched those of medium spiny TI + GG neurons (fig. 5a, b). There were no signs of inadequate metal impregnation in these cells, although it was sometimes difficult to distinguish singly labeled medium aspiny neurons from medium spiny GG neurons in F52 kittens. The characteristic of sparse dendritic spines did not preclude double labeling, as shown by TI + GG neurons in adult cerebral cortex with nonpyramidal small-to-medium cell bodies and aspiny dendrites.

Additionally, 5 large aspiny TI + GG neurons from 4 cases were ovoidal multipolar cells with long somatic diameters $\geq 25 \mu \mathrm{m}$, thick dendritic shafts with calibers of $\geq 2-4 \mu \mathrm{m}$, and sparse dendritic spines dispersed throughout the dendritic arbors. They had less dendritic branching, longer dendritic segments and larger dendritic arbors than medium spiny or medium aspiny TI + GG neurons (fig. $5 \mathrm{a}-\mathrm{c}$ ). These neurons were site specific, since comparable large aspiny neurons singly labeled for GG never colocalized TI in the adjacent globus pallidus. Upon close scrutiny of our developmental reference materials, infrequent instances (10-20 cells/case) of singly labeled large neostriatal TI neurons, with somatic diameters approximately twice those of principal medium TI neurons at the same age, were evident in most cases $\geq \mathrm{F} 30$. These neurons were not overlaid profiles of medium TI cells, but resembled large Nissl-stained neurons settled in the neostriatal mantle zone.

\section{Discussion}

The results of this investigation support 3 novel conclusions. (1) Colocalization of immunohistochemical and GG labels is a feasible, rational and productive means to identify and determine the somatodendritic characteristics and morphogenesis of neurons with interesting neu- 
rochemical signatures. (2) Application of this method to developing feline neostriatum demonstrates that the principal tachykinin cells are medium spiny neurons, which undergo progressive growth of cell bodies, dendritic arbors and dendritic spines during the late fetal and postnatal periods. (3) Concordance between tachykinin and medium spiny neuronal phenotypes is strong but incomplete because rare medium and large aspiny neurons also express tachykinins.

The somatodendritic phenotypes of medium tachykinin neurons in feline neostriatum match tachykinin cells of unknown somatodendritic phenotypes commonly reported in mammals [1-3, 6-9]. Principal and minor variants of medium spiny tachykinin neurons are identical to medium spiny neurons with unknown neuromodulator signatures shown by Golgi impregnation, which constitute approximately $95 \%$ of neurons in adult feline neostriatum [19]. They resemble respectively the medium spiny type 1 and 2 neurons of monkey neostriatum [20], but are considered to represent a single phenotype because the variant has little known functional significance. Our results replicate, extend and complement previous discoveries of both forms based on combined peroxidase immunohistochemistry and GG in Izzo et al. [12]. They use stringent fixation and dense gold toning to obtain a small sample of doubly labeled cells for correlative light and electron microscopy. Precise label localization and detection of synaptic junctions (an important characteristic of mature neuronal identity) are achieved at the expense of a sufficient sample of somatodendritic variation in the population of tachykinin neurons. We used different fixation and photoreduction procedures to obtain a much larger sample of doubly labeled cells for a morphogenetic survey by light microscopy. A credible assessment of range of somatodendritic variation and accurate label recognition is achieved at the expense of precise label localization; however, subcellular localizations and optical properties of labels are similar in both reports. In contrast to results obtained by alternative methods based on combinations of fluorescent and biocytin labels, the technical advantages of these tissue preparations consist of near-permanent durability for extended light-optical examination, superior sensitivity of indirect peroxidase-antiperoxidase immunohistochemical methods for neuropeptide detection, high contrast resolution of fine structural details such as the thin spine necks of immature neurons and typical isolation of individually labeled cells. The numerical predominance of medium spiny tachykinin neurons is consonant with the notion that they must produce most of the substance $\mathrm{P}$, neuroki- nin $A$ and neurokinin $B$ in neostriatum, a persistent issue in the compartmentalized 'striosomal' organization of basal ganglia $[5,21,22]$.

Unusual phenotypes of large and medium aspiny tachykinin neurons in feline neostriatum are congruent with observations of large tachykinin cells [10, pers. observations] and the indirect inference of medium aspiny tachykinin neurons [11]. These phenotypes are identical to large and medium 'smooth dendrite' (aspiny) neurons, which constitute approximately $3-4 \%$ of neurons in adult feline neostriatum [19]. Low frequencies and inconstant detection across cases suggest that they are minor subsets $(<10-20 \%)$ of these small populations of large and medium aspiny neurons, cells with characteristically high levels of substance $P$ receptor expression [23]. Their functional significance is likely to be modest. However, they are a conceptually important demonstration that the coupled expression of neuromodulator and somatodendritic characteristics in neostriatal neurons is heavily weighted toward, but not absolutely committed to, the generation of 1 complex phenotype. Similar slight diversities of somatodendritic phenotypes, including the minor variant of the principal medium spiny phenotype, occur among GABA-synthesizing and striatonigral projection neurons $[24,25]$. These more complex combinations of characteristics could support both early nonsynaptic and later synaptic cellular interactions in basal ganglia [2]. Tachykinins remain undetected in small populations of small 'smooth dendrite' and medium 'varicose dendrite' aspiny neurons [19], perhaps the only somatodendritic phenotypes devoted exclusively to local axonal connections within neostriatum. The near-constant proportions of the heterogeneous phenotypes of tachykinin neurons are consistent with an important but largely unvoiced assumption of an elemental stability within the various cellular compartments of 'striosomal' organization [5]. After the inception of tachykinin phenotypes during development, levels of tachykinin expression may exhibit periodic regulation, but the characteristic tachykinin identity appears to be permanent. This suggestive but inconclusive evidence does not preclude the simultaneous expression of multiple modulator and modulator/transmitter identities by neostriatal neurons $[3,7]$.

Progressive growth of cell bodies, dendrites and dendritic spines is the foremost morphogenetic process found in the age sequence of medium spiny tachykinin neurons in late fetal and postnatal feline neostriatum. Postnatally, these neurons have the same maturational changes as Golgi-impregnated medium spiny neurons [26]. Prena- 
tally, their dendritic outgrowth by branch addition decelerates in coincidence with the eruption of dendritic spines and initiation of synaptogenesis of gray type I corticostriatal and thalamostriatal afferents [27]. Phenotypic characteristics of neuronal size and dendritic spine generation appear to be early-determined, intrinsic properties of target cells in this interaction, since large and medium aspiny tachykinin neurons retain essentially smooth dendrites despite continued shaft outgrowth during this period.

Immature brain is a traditional focus for Golgi impregnation due to the central importance of morphogenesis for the doctrine of synaptic connectivity, more comprehensive staining of small young cells and extensive staining of axons prior to myelination. However, it is difficult to pursue these studies in a meaningful manner before the differentiation of adult-like, heterogeneous somatodendritic phenotypes. Our survey of TI + GG colocalization in neostriatal neurons, with its perhaps unexpected interest in persistent, mature phenotypes, has significant implications for a resolution of this developmental problem. The morphogenetic sequence and outcome for the principal phenotype of medium spiny tachykinin neurons is now demonstrated beyond a reasonable doubt by positive evidence (i.e. direct observations from substantial samples of doubly labeled cells). Distinct phenotypes of medium spiny and aspiny neurons cannot be distinguished before preferential spine generation on dis- tal dendrites near F52, and the TI + GG method cannot be applied to earlier stages of development due to technical difficulties. Fortunately, these limits do not preclude earlier structural analyses. The pattern of progressive morphogenesis, low levels of nonpreferential background apoptosis and predominance of medium spiny tachykinin neurons after F52 indicates that medium tachykinin cells detected as early as F30 are already destined to express a persistent medium spiny phenotype, as demonstrated to a reasonable certainty by positive evidence (i.e. such phenotype identifications would, by necessity, be accurate for $\geq 99 \%$ of tested cells). The small population of large tachykinin cells destined to express a large aspiny phenotype is also established as early as F30. The only source of uncertainty is the small population of medium tachykinin cells destined to express a medium aspiny phenotype. Thus, the early somatodendritic development of cells committed to the medium spiny tachykinin fate can be reasonably well delineated by Golgi impregnations of their structural surrogates, medium-sized neostriatal neurons, prior to F52.

\section{Acknowledgements}

This work was supported by the United States Public Health Service grants HD05958 and NS24596.

\section{References}

$>1$ Diez-Guerra FJ, Veira JA, Augood S, Emson PC: Ontogeny of the novel tachykinins neurokinin A, neurokinin B and neuropeptide $\mathrm{K}$ in the rat central nervous system. Reg Peptides 1989;25:87-97.

-2 Boylan MK, Levine MS, Buchwald NA, Fisher RS: Patterns of tachykinin expression and localization in developing feline neostriatum. J Comp Neurol 1990;293:151-163.

3 Penny GR, Afsharpour S, Kitai ST: The glutamate decarboxylase-, leucine enkephalin-, methionine enkephalin- and substance Pimmunoreactive neurons in the neostriatum of the rat and cat: evidence for partial population overlap. Neuroscience 1986;17:10111045.

4 Maggio JE: Tachykinins. Annu Rev Neurosci 1988;11:13-28.

$\checkmark 5$ Gerfen CR: The neostriatal mosaic: multiple levels of compartmental organization in the basal ganglia. Annu Rev Neurosci 1992;15: 285-320.

Morphogenesis of Neostriatal Tachykinin Neurons
6 Kohno J, Shiosaka S, Shinoda K, Inagaki S, Tohyama M: Two distinct strio-nigral substance $P$ pathways in the rat: an experimental immunohistochemical study. Brain Res 1984;308:309-317.

7 Beckstead RM, Kersey KS: Immunohistochemical demonstration of differential substance $\mathrm{P}$, met-enkephalin-, and glutamic acid decarboxylase-containing cell body and axon distribution in the corpus striatum of the cat. J Comp Neurol 1985;232:481-498.

$>8$ Harlan RE, Garcia MM, Krause JE: Cellular localization of substance P- and neurokinin A-encoding preprotachykinin mRNA in the female rat brain. J Comp Neurol 1989;287: 179-212.

-9 Marksteiner J, Saria A, Krause JE: Comparative distribution of neurokinin B-, substance $\mathrm{P}$ - and enkephalin-like immunoreactivities and neurokinin B messenger RNA in the basal forebrain of the rat: evidence for neurochemical compartmentation. Neuroscience 1992;51:107-120.
10 Ljungdahl A, Hokfelt T, Nilsson G: Distribution of substance P-like immunoreactivity in the central nervous system of the rat. I. Cell bodies and nerve terminals. Neuroscience 1978;3:861-943.

11 Bolam JP, Somogyi P, Takagi H, Fodor I, Smith AD: Localization of substance P-like immunoreactivity in neurons and nerve terminals in the neostriatum of the rat: a correlated light and electron microscopic study. J Neurocytol 1983;12:325-344.

12 Izzo PN, Graybiel AM, Bolam JP: Characterization of substance P- and [met] enkephalinimmunoreactive neurons in the caudate nucleus of cat and ferret by a single section Golgi procedure. Neuroscience 1987;20:577-587.

13 Sternberger LA: Immunohistochemistry. Englewood Cliffs, Prentice Hall, 1986.

14 Cuello AC, Galfre G, Milstein C: Detection of substance $P$ in the central nervous system by a monoclonal antibody. Proc Natl Acad Sci USA 1979;76:3532-3536. 
15 Itto K, Konishi S, Nomura N, Mizuho Y, Nakamura Y, Sugimoto T: Application of coupled oxidation reaction to electron microscopic demonstration of horseradish peroxidase: cobalt-glucose oxidase method. Brain Res 1980;195:29-36.

16 Gabbot PL, Somogyi J: The 'single' section Golgi-impregnation procedure: methodological description. J Neurosci Methods 1984;11:221-230.

17 Fairen A, Peters A, Saldanha J: A new procedure for examining Golgi-impregnated neurons by light- and electron-microscopy. J Neurocytol 1977;6:311-337.

18 Wouterlood FG: Techniques for converting Golgi precipitate in CNS neurons into stable electron microscopic markers. Microsc Res Tech 1992;23:275-288.
19 Kemp JM, Powell TP: The structure of the caudate nucleus of the cat: light and electron microscopy. Philos Trans R Soc Lond (Biol) 1971;262:429-439.

20 DiFiglia M, Pasik P, Pasik T: A Golgi study of neuronal types in the neostriatum of monkeys. Brain Res 1976;114:245-256.

21 Breur O, Lawhorn C, Miller T, Smith DM, Brown LL: Functional architecture of the mammalian striatum: mouse vascular and striosome organization and their anatomic relationships. Neurosci Lett 2005;385:198203.

22 Kemmel ML, Jabourian M, Perez S, Glowinski J: Peptidergic regulation of cholinergic transmission in the dorsal striatum; in Bolam JP, Ingham CA, Magill PJ (eds): Peptides and Acetylcholine in the Striatum. New York, Springer US, 2006, pp 119-128.

23 Shigemoto R, Nakaya Y, Nomura S, OgawaMeguro R, Ohishi H, Kaneko T, Nakanishi S, Mizuno N: Immunocytochemical localization of rat substance $\mathrm{P}$ receptor in the striatum. Neurosci Lett 1993;153:157-160.
24 Fisher RS, Buchwald NA, Hull CD, Levine MS: Neurons of origin of striatonigral axons in the cat: connectivity and Golgi markers of somatodendritic architecture. Brain Res 1986;397:173-180.

25 Fisher RS: The anatomy of GABAergic neostriatal neurons: medium-sized spiny cells are the principal inhibitory neurons in the basal ganglia. Anat Rec 1988;220:37A.

26 Hull CD, McAllister JP, Levine MS, Adinolfi AM: Quantitative developmental studies of feline neostriatal spiny neurons. Dev Brain Res 1981;1:309-332.

27 Fisher RS, Levine MS, Adinolfi AM, Hull $\mathrm{CD}$, Buchwald NA: The morphogenesis of glutamic acid decarboxylase in the neostriatum of the cat: neuronal and ultrastructural localization. Dev Brain Res 1987;33:215-234. 